\title{
Dall'«unité artificielle» al «fond du cœur» Manzoni lettore di Diderot
}

\section{Bianca Gai}

\section{(2) OpenEdition}

1 Journals

\section{Edizione digitale}

URL: http://journals.openedition.org/studifrancesi/8160

DOI: 10.4000/studifrancesi.8160

ISSN: 2421-5856

\section{Editore}

Rosenberg \& Sellier

\section{Edizione cartacea}

Data di pubblicazione: 1 mai 2009

Paginazione: 120-133

ISSN: 0039-2944

\section{Notizia bibliografica digitale}

Bianca Gai, «Dall'«unité artificielle» al «fond du cœur» Manzoni lettore di Diderot», Studi Francesi [Online], 157 (LIII | I) | 2009, online dal 30 novembre 2015, consultato il 09 janvier 2021. URL: http:// journals.openedition.org/studifrancesi/8160 ; DOI: https://doi.org/10.4000/studifrancesi.8160

\section{(c) (i) $\odot$}

Studi Francesi è distribuita con Licenza Creative Commons Attribuzione - Non commerciale - Non opere derivate 4.0 Internazionale. 


\section{Dall'«unité artificielle»al «fond du coeur» Manzoni lettore di Diderot}

Se noi inventassimo ora una storia a bel diletto, ricordevoli dell' acuto e profondo precetto del Venosino, ci guarderemmo bene dal riunire due immagini così disparate come quelle che si associavano nella mente di Fermo [una treccia nera e una barba bianca]; ma noi trascriviamo una storia veridica; e le cose reali non sono ordinate con quella scelta, né temperate con quella armonia che sono proprie del buongusto: la natura e la bella natura sono due cose diverse ${ }^{1}$.

Così, nel Fermo e Lucia, Manzoni istituiva una radicale dicotomia fra l'andamento armonico prescritto agli eventi dalla convenzione letteraria e la dissonanza avvertibile nelle «cose reali», disordinate dalla coincidenza paradossale di immagini «disparate». Analoga discrepanza aveva già segnalato, secondo simili movenze, Diderot, che in Jacques le fataliste distingueva la disposizione disarmonica della «chose comme elle s'est passée» dall'unité imposta alle vicende dalle regole letterarie:

LE MAITTRE. Notre hôtesse, vous narrez assez bien; mais vous n'êtes pas encore profonde dans l'art dramatique. Si vous vouliez que cette jeune fille intéressât, il falloit lui donner de la franchise, et nous la montrer victime innocente et forcée de sa mère et de la Pommeraye [...] il falloit préparer ainsi le raccommodement de cette femme avec son mari. Quand on introduit un personnage sur la scène, il faut que son rôle soit un; or je vous demanderai, notre charmante hôtesse, si la fille qui complote avec deux scélérates est bien la femme suppliante que nous avons vue aux pieds de son mari. Vous avez péché contre les règles d'Aristote, d'Horace, de Vida et de Le Bossu.

L'HÔTESSE. Je ne connois ni bossu ni droit, je vous ai dit la chose comme elle s'est passée, sans en rien omettre, sans y rien ajouter. Et qui sait ce qui se passoit au fond $d u$ coeur de cette jeune fille, et si, dans les momens où elle nous paroissoit agir le plus lestement, elle n'étoit pas secrètement dévorée de chagrin? ${ }^{2}$

(1) A. Manzoni, Fermo e Lucia, a cura di S. S. Nigro, Milano, Mondadori, 2002, III, VIII, pp. 582-583 (da ora in poi si indicherà l'edizione con la sigla $F L$ ).

(2) D. Diderot, Jacques le fataliste, in ID., Euvres de Denis Diderot, publiées sur les manuscrits de l'auteur par Jacques-André Naigeon de l'Institut National des Sciences, à Paris, chez Deterville libraire, rue du Battoir, n. 16, an VIII [1800], vol. XI, pp. 236-237; corsivi miei. Si citerà da ora in poi da questa edizione, indicandola con la sigla $J F$, riproducendone la grafia settecentesca e aggiungendo, per comodità del lettore, la numerazione di pagina dell'edizione più recente, consultabile in D. DIDEROT, Euvres, a cura di L. VersinI, 5 voll., Paris, Laffont, 1994, vol. II, Contes (qui la citazione si legge a p. 825). Nell'analisi della consistenza di un intertesto diderotiano nell'opera di Manzoni, la critica si è arrestata sostanzialmente al confronto tematico fra l'episodio della monaca di Monza dei Promessi Spo- si e le dinamiche narrative della Religieuse. Ancora da sondare è la possibilità, aperta per la prima volta da Giovanni Getto in una nota del suo Manzoni europeo, che la conoscenza del philosophe da parte di Manzoni risultasse più ampia e complessa. Tutta da verificare è inoltre la plausibilità storico-documentaria di tale ipotesi, considerando la circolazione tarda e la fortuna recente di molta della produzione di Diderot (cfr. G. GETTO, I capitoli «francesi», in ID., Manzoni europeo, Milano, Mursia, 1971, pp. 59-140). Prima di lui si sono occupati del rapporto Manzoni-Diderot i seguenti studi: A. LuZIO, Manzoni e Diderot. La monaca di Monza e "La Religieuse", Milano, Dumolard, 1884; L. F. BenEDETTO, Sorelle francesi di Gertrude, in «Marzocco», XXXVI, 1931; M. D. Busnelui, Per la genesi della «Signora di Monza», in «Atti del Reale Istituto Veneto di scienze, lettere ed arti», XVII, 1932-1933, parte II, pp. 849-874; L. Russo, Manzoni poeta e Diderot oratore, in ID., Ritratti e disegni storici: da Machiavelli 
Il contrasto cromatico che delinea il pensiero di Fermo funziona in maniera analoga all'incongruenza logica generata, nel conte diderotiano, dal comportamento della «jeune fille» narrato dall'ostessa: alla compiutezza delle regole classiche la scrittura romanzesca oppone la disarmonia non prevedibile dell'associazione mentale e la bizzarria delle passioni individuali. «Mente» di Fermo e «fond du cœur» di Mlle d'Aisnon si corrispondono in quanto elementi di negazione di precetti che alludono a un ambito teatrale, anche se in maniera meno evidente nel Fermo e Lucia che nel passo di Diderot. Se l'«art dramatique» di ispirazione aristotelica campeggia infatti immediatamente in Jacques le fataliste come codice di riferimento ineludibile per il maître, inducendolo ad applicare a una narrazione le categorie teatrali di «scène» e «rôle», nessun accenno esplicito al teatro emerge dalla notazione manzoniana. Altrove tuttavia, all'interno della riflessione sullo statuto del romanzo delle lettere a Fauriel, Manzoni adotta nell'argomentare il suo rifiuto dell'esprit romanesque la stessa terminologia della trattazione antiaristotelica della Lettre à M. Chauvet, così come Diderot si era valso di un formulario preso a prestito dalla drammaturgia per tracciare un'analisi estetica della narrazione romanzesca dell'bôtesse:

Dans tous les romans que j'ai lus, il me semble de voir un travail pour établir des rapports intéressans et inattendus entre les différens personnages, pour les ramener sur la scène de compagnie, pour trouver des événemens qui influent à-la-fois et en différente manière sur la destinée de tous, enfin une unité artificielle que l'on ne trouve pas dans la vie réelle. Je sais que cette unité fait plaisir au lecteur, mais je pense que c'est à cause d'une ancienne habitude; je sais qu'elle passe pour un mérite dans quelques ouvrages qui en ont un bien réel et du premier ordre, mais je suis d'avis qu'un jour ce sera un objet de critique: et qu'on citera cette manière de nouer les événemens, comme un example de l'empire que la coutume exerce sur les esprits les plus libres et les plus élevés, ou des sacrifices que l'on fait au goût établi³

Coincide nei due autori la riconduzione del principio di unità di eventi e comportamenti all'ambito artificioso di una scène teatrale: come Diderot, Manzoni identifica le regole da cui la scrittura romanzesca rifugge con le convenzioni sancite dalle poetiche drammaturgiche di ispirazione aristotelica. In entrambi, l'abbandono del teatro conduce alla predilezione del genere narrativo, che nella sua assenza di codificazione si rivela più adatto ad accogliere l'apertura al vero che era stata inizialmente pensata per il genere drammatico. Ma per lo stesso motivo il teatro rappresenta un orizzonte normativo cui continuamente il testo romanzesco allude, tentando ovunque di rovesciarne i postulati ${ }^{4}$.

L'attenzione di Manzoni alla produzione drammaturgica e narrativa di Diderot è in linea con il cambiamento delle modalità di ricezione del philosophe verificatosi proprio negli ambiti culturali di riferimento per la sua formazione intellettuale. A

a Carducci, Bari, Laterza, 1937, pp. 242-259. Più recentemente è tornato sull'argomento l'articolo di G. GogGI, Ancora su Manzoni-Diderot: rapporti intertestuali e schemi narrativi comuni, in «Linguistica e letteratura», XI, 1-2, 1986, pp. 47-90.

(3) A. Manzoni, Carteggio Manzoni-Fauriel, a cura di I. BOTTA, in Edizione nazionale ed europea delle opere di Alessandro Manzoni, a cura di G. VIGorelli, Milano, Centro Nazionale di Studi Manzoniani, 2000-2005, vol. 27 (2000), lettera del 29 maggio 1822, p. 353; corsivi miei.

(4) Gilberto Lonardi ha posto in luce i movimenti speculari di «romanzizzazione del tragico» e di «interferenza dell'esperienza tragica [...] col narrare manzoniano», individuando per alcuni temi dei Promessi Sposi una «traslazione» dal formulario di luoghi tragici manzoniani (il cui esempio più significativo è la derivazione del traditore comico don Abbondio dal traditore tragico Marco) e nelle opere tragiche la sperimentazione della diffrazione del punto di vista che animerà il romanzo (cfr. G. LONARDI, Ermengarda e il pirata: Manzoni, dramma epico, melodramma, Bologna, il Mulino, 1991). Nella poesia tragica manzoniana si individua già l'«esistenza di molteplici piani di azione, non necessariamente connessi alle peripezie e cioè allo sviluppo dell'intreccio», che caratterizzerà poi il romanzo (cfr. P. Bosisio, Manzoni drammaturgo dal "Carmagnola" all' "Adelchi", in «Otto/Novecento», X, 2, 1986, pp. 5-37). 
sancire l'imprimatur alla circolazione europea del teatro di Diderot era stato Gotthold Ephraïm Lessing, licenziandone nel 1760 una traduzione tedesca (Das Theater des Herrn Diderot) e valendosi delle teorie dell'illuminista francese a suffragio della polemica contro le regole aristoteliche condotta nella Hamburgische Dramaturgies. L'apprezzamento di Lessing non sarà sfuggito al Manzoni lettore della Dramaturgie e grande estimatore del Laokoon ${ }^{6}$, anche perché riverberarono subito in Italia i riflessi dell'interpretazione lessinghiana. Uscirono infatti precocemente le traduzioni del Père de famille e del Fils naturel e il «Caffè» illustrò, in un articolo di Pietro Secchi, le novità della poetica drammaturgica diderotiana soffermandosi, in consonanza alla lettura della Hamburgische Dramaturgie, sulle innovazioni più in contrasto con gli automatismi aristotelici del teatro classico francese ${ }^{8}$. Ma la grande intuizione dei lettori tedeschi di Diderot fu la scoperta della modernità dei contes philosphiques e dei saggi di estetica. Nel 1785, data di poco successiva a quella in cui il diario di Goethe registra l'avvenuta lettura di Jacques le fataliste, Friedrich Schiller tradusse l'episodio

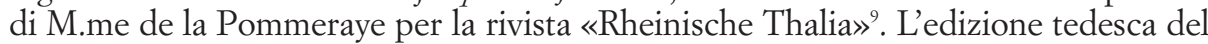
romanzo, uscita a Berlino nel 1792 con il titolo Jakob und sein Herr, precedette di quattro anni la prima edizione francese e nel 1805 Jacques le fataliste fu presentato dal Dialogo sulla poesia di Friedrich Schlegel come esempio di «arabesco», genere esemplato sul modello del Tristram Shandy sterniano che era ritenuto dal critico una sorta di anticipazione del romanzo totale inteso in senso romantico ${ }^{10}$. A dirigere il gusto europeo sono soprattutto, però, le scelte traduttive di Goethe: furono la sua

(5) R. HEITNER, Lessing's indebtedness to Diderot, in «MLN», LXV, 1950, pp. $82-88$ e J. CHOUILLET, Diderot vu par Lessing, in AA. Vv., Diderot et le théâtre, Paris, Comédie Française, 1984, pp. 29-30.

(6) Cfr. F. ForTI, Lessing e la poetica drammatica del Manzoni, in ID., Lo stile della meditazione: Dante, Muratori, Manzoni, Bologna, Zanichelli, 1981, pp. 146-159. Per le edizioni possedute da Manzoni, cfr. «Annali manzoniani», VI, 1981, pp. 114-209.

(7) Il Padre di famiglia. Commedia in cinque atti in prosa del celebre signor Diderot, tradotta dal francese in italiano dal signor Michele Bocchini, Livorno, G. P. Fanteschi, 1762; Diderot, Il figlio naturale, ossieno le pruove della virtù. Commedia, Modena, Stamperia Montanari, 1768 (cfr. M. D. BusNelli, Essai bibliographique, in ID., Diderot et l'Italie, Paris, Champion, 1925).

(8) L'articolo si intitola Del teatro (cfr. Il caffè [1764-1766], a cura di G. Francioni e S. RomaGNOLI, Torino, Bollati Boringhieri, 1993) e si sofferma sulla tecnica delle «scènes simultanées», sulla nuova categorizzazione dei generi, che interponeva il genere serio fra il tragico e il comico, e sull'uso della pantomima. La predilezione di Secchi per Diderot è in consonanza con la linea della rivista, il cui fondatore Pietro Verri vi scriveva di teatro apprezzando in particolar modo il dramma borghese (cfr. S. Romagnoli, Il teatro e il «Caffé», in ID., La buona compagnia. Studi sulla letteratura italiana del Settecento, Milano, Angeli, 1983, pp. 158-177). Per la diffusione in Italia del dramma borghese e in particolare della teoria drammatica di Diderot, cfr. S. GugenHeIM, Drammi e teorie drammatiche del Diderot e loro fortuna in Italia, in «Études italiennes», 3, 1921, pp. 27-35 e E. Levi Malvano, $L a$ fortuna d'una teoria drammatica in Italia, in «Gior- nale storico della letteratura italiana», XIII, 1935, pp. 60-103.

(9) Cfr., anche per gli autori tedeschi che seguono nella trattazione, R. MORTIER, Diderot en Allemagne, Paris, Presses Universitaire de France, 1954 e, per Shiller, O. W. Johnston, Schiller, Diderot and the Dalberg manuscript, in «The Germanic Review», XLVI, 3, 1971, pp. 167-181. Per il rapporto con Manzoni, cfr. G. GüNTER, Manzoni, Scbiller e A. W. Scblegel: dalla teoria degli stili all'episodio della madre di Cecilia, in «Esperienze letterarie», XXVIII, 1, 2003, pp. 13-34.

(10) Cfr. F. Schlegel, Dialogo sulla poesia, in Frammenti e scritti di estetica, trad. it., Firenze, Sansoni, 1967, p. 209. Il dialogo fu pubblicato nella rivista «Athenaeum» (vol. III, 1800) e fu poi ristampato a cura dello stesso Schlegel nei Sämmtliche Werke, usciti a Vienna nel 1823. Di Schlegel si conserva nella biblioteca manzoniana di Brusuglio il volume Storia della letteratura antica e moderna, nella traduzione di F. Ambrosoli datata 1828, in cui si elogia il «geniale Diderot», protagonista con Voltaire e Rousseau di una rivoluzione delle forme romanzesche: «Il romanzo dovette diventar principalmente il genere favorito per quelli il cui entusiasmo di natura non trovava modo di esprimersi nelle forme antiche; perrocchè questa forma, se tale si può chiamare, era libera da tutte le catene, alle quali altrimenti dovevasi inevitabilmente soggiacere nella poesia [...]. Voltaire, Rousseau e Diderot si servirono adunque spesso dei romanzi assai arbitrariamente, e solo siccome una forma ove deporre certe idee proprie per le quali nessun'altra forma era meglio acconcia» (F. SCHLegel, Storia della letteratura antica e moderna, nella versione di F. AmBrosoli [1828], a cura di R. Assunto, Paravia, 1974, pp. 272-274). 
versione tedesca degli Essais sur la peinture, comparsa sulla rivista «Propyläen» nel 1799, e la sua traduzione del Neveu de Rameau, edita nel 1805 con il titolo Rameaus Neffe, a rivelare il Diderot teorico estetico e il Diderot narratore ${ }^{11}$.

Anche l'altro possibile diaframma di intermediazione nel contatto di Manzoni con l'opera diderotiana, il milieu degli idéologues da lui frequentato durante i soggiorni parigini giovanili ${ }^{12}$, riscoprì e reinterpretò l'opera dell'ispiratore Diderot secondo modalità del tutto in sintonia con i meccanismi della ricezione tedesca ${ }^{13}$. La rivista organo di quel movimento, la «Décade philosophique» ${ }^{14}$, oltre a pubblicare un'interpretazione molto favorevole della Religieuse e tre articoli riguardanti gli Essais sur la peinture, concesse ampia risonanza nel 1796 al recupero da parte dell'Institut de France di una copia in tedesco di Jacques le fataliste, si può dire fino ad allora inedito, e fornì notizia degli accordi con il principe di Prussia depositario della copia, ospitando una recensione dell'edizione del conte uscita in quell'occasione, accompagnata da alcuni stralci dell'opera ${ }^{15}$. A testimonianza della possibilità che Manzoni abbia subito l'influenza dell'interpretazione di Diderot restituita dagli intellettuali del SetteOttocento tedesco resta una traccia nella biblioteca manzoniana di Via Morone. La raccolta Contes moraux et nouvelles idylles de D. et Salomon Gessner ${ }^{16}$ qui conservata, che contiene in pendant agli idilli gessneriani due racconti di Diderot (Les deux amis de Bourbonne e Entretien d'un père avec ses enfants), è una traduzione francese del vo-

(11) La traduzione-commento, dal titolo Diderots Versuch über die Malerei, apparve su «Propyläen» nel 1799 (si può leggere in traduzione italiana in J.W. GOETHE, Scritti sull' arte e sulla letteratura, trad. it., a cura di S. ZECCHI, Torino, Bollati Boringhieri, 1992, pp. 119-157). Per il rapporto di Goethe con Manzoni, cfr. M. PuPpo, Manzoni e Goethe: il dibattito su poesia e storia, in ID., Poesia e verità: interpretazioni manzoniane, Messina-Firenze, D'Anna, 1979, pp. 23-40; Goethe e Manzoni: rapporti tra Italia e Germania intorno al 1800, Atti del colloquio Goethe e Manzoni tenutosi alla Villa Vigoni di Menaggio (Como) nell'aprile 1988, Firenze, Olschki, 1992; C. LERI, Manzoni e la littérature universelle, Milano, Casa del Manzoni, 2002. La biblioteca di Via Morone possiede un'edizione delle opere complete di Goethe: Goethe's Werke, 40 voll., Stuttgart und Tübingen, J. G. Cotta, 1827-1831 (cfr. «Annali manzoniani», VI, 1981, p. 104).

(12) Per gli influssi dell'idéologie nel pensiero manzoniano, cfr. E. GABBUTI, Il Manzoni e gli ideologi francesi, Firenze, Sansoni, 1936; M. DAVID, Manzoni et l' «idéologie», in «Revue des études italiennes», XXXII, 1-4, 1986, pp. 42-77; P. GiannANTONIO, Alla scuola del Manzoni, Torino, Genesi, 1989, in particolare il capitolo Alla scuola degli ideologi, pp. 9-29; L. BADINI CONFALONIERI, La "Lettre à M. Chauvet", les «affections sympathiques» et la leçon de Cabanis, in «Revue des études italiennes», XLIX, 3-4, 2003, pp. 273-295 e ID., Les régions de l'aigle et autres études sur Manzoni, Berne, Peter Lang, 2005.

(13) Il cenacolo degli idéologues, e soprattutto Fauriel, intratteneva fitti scambi con il mondo tedesco (cfr. M. EsPaGNE, Claude Fauriel en quête d'une méthode, ou l'Idéologie à l'écoute de l'Allemagne, in «Romantisme», 73, 1991, pp. 7-17).

(14) Spesso la «Décade» condusse una sorta di rivalutazione apologetica di Diderot, rendendone noti inediti di contenuto politico e letterario e pubblicando interventi encomiastici sul suo conto, come l'Éloge philosophique de Denis Diderot, pronunciato nel 1800 all'Institut National da Joseph Baconière de Salverte, o la difesa di Pierre-Louis Ginguené, che proprio nell'anno in cui Manzoni giungeva per la prima volta a Parigi confutava gli attacchi al philosophe di La Harpe, all'interno del più ampio progetto di opposizione all'opera del celebre critico dal titolo La Pbilosophie du XVIII ${ }^{\circ}$ siècle. Per la relazione fra idéologues e philosophes, cfr. S. Moravia, Gli «idéologues» e l'età dei lumi, in «Belfagor», XXVIII, 3, 1973, pp. 254-259 e ID., Il pensiero degli idéologues, Firenze, La Nuova Italia, 1974.

(15) Per la ricostruzione dei riferimenti della «Décade» a Diderot si sono considerati i seguenti interventi, a cui si rimanda: A. FREER, "Jacques le fataliste" e "La Religieuse" nelle testimonianze critiche contemporanee alla loro pubblicazione, in Saggi e ricerche di letteratura francese, 3 voll., Milano, Feltrinelli, 1963, vol. III, pp. 67-103; J. KITCHIN, La «Décade» (1794-1807): un journal «philosophique», Paris, Minard, 1965 e M. RÉGALDO, La «Décade» et les philosophes, in «Dix-huitième siècle», 2, 1970. Cfr. anche l'antologia a cura di J. BAULAD-AYOUB, La «Décade philosophique» comme système, 5 voll., Mayenne, Presses Universitaires de Rennes, 2003.

(16) Contes moraux et nouvelles idylles de D. et Salomon Gessner, à Zuric, chez l'auteur, 1773. La raccolta ha destato un certo interesse nei critici, cfr. R. MorTiEr, Diderot en Allemagne, cit., p. 184 e R. Marchal, Des satyres parmi les nymphes: "Contes moraux et nouvelles Idylles" de Diderot et de Salomon Gessner, in «Travaux de littérature», XIV, 2001, pp. 309-319. 
lume che, uscito in Germania l'anno prima con il titolo Moralische Erzäblungen und Idyllen von Diderot und S. Gessner, aveva destato l'interesse della cultura tedesca per la produzione dell'illuminista, tanto che Goethe aveva inserito nella sua autobiografia un commento senza dubbio riferibile ai racconti diderotiani presenti nella raccolta ${ }^{17}$. È significativo che l'unica opera diderotiana presente in una biblioteca manzoniana, eccettuata l'Encyclopédie, sia la stessa ad aver innescato l'attenzione dell'area tedesca per le capacità narrative del philosophe, che invece rimanevano in Francia secondarie rispetto alla sua attività filosofica ed enciclopedica. Gli scrittori tedeschi, a partire da Lessing, vedevano in Diderot il primo contestatore del teatro classico francese e il primo ideatore di un modus narrativo aperto a temi e personaggi sconosciuti al romanzo francese del Settecento. Ed è esattamente a livello di poetica drammatica e di poetica romanzesca, e soprattutto della loro reciproca connessione e derivazione, che si rintracciano i punti di tangenza fra Manzoni e Diderot.

Il rifiuto delle unità aristoteliche della Lettre à $M$. Chauvet sembra presupporre una conoscenza diretta della drammaturgia diderotiana, a partire dall'assimilazione fra condanna estetica delle regole letterarie e condanna filosofica della tortura, entrambe considerate momenti necessari di una più generale guerra contro l'errore:

La torture a duré long-temps encore après l'immortel traité des délits et des peines; cela reconnu, il faudrait être bien impatient et bien égoïste pour se plaindre de la ténacité des préjugés littéraires [...]. L'erreur est déjà troublée dans sa possession, avec le temps elle sera dépossédée ${ }^{18}$.

Il faut espérer que quelque jour un homme de génie sentira l'impossibilité d'atteindre ceux qui l'ont précédé dans une route battue, et se jettera de dépit dans une autre; c'est le seul événement qui puisse nous affranchir de plusieurs préjugés que la philosophie a vainement attaqués ${ }^{19}$.

Nel dettato diderotiano, la liberazione dalle regole trovava un parallelo nell'ampliamento dei contenuti, estesi fino a sfidare l'attesa convenzionale del pubblico:

Mais admirez la bizzarrerie des peuples policés. La délicatesse y est quelquefois poussée au point, qu'elle interdit à leur poëtes l'emploi des circonstances même qui sont dans leurs mœurs, et qui ont de la simplicité, de la beauté et de la vérité [...]. O peuple plaisant et léger! Quelles bornes vous donnez à l'art! Quelle contrainte vous imposez à vos artistes! Et de quels plaisirs votre délicatesse vous prive! [...] Il faut que le goût d'un peuple soit incertain; lorsqu'il admettra dans la nature, des choses dont il interdira l'imitation à ses artistes, ou lorsqu'il admirera dans l'art des effets qu'il dédaigneroit dans la nature ${ }^{20}$.

Anche il concetto di «délicatesse», corrispondente psicologico della «règle», riemerge invariato (talvolta declinato come «susceptibilité») nella riflessione manzoniana, a indicare un'abitudine percettiva del pubblico che una poetica orientata al vero ha il dovere di disattendere:

(17) «I suoi figli della natura che egli sapeva far risaltare e nobilitare con grande arte oratoria, ci andavano molto a genio, i suoi baldi cacciatori di frodo e contrabbandieri ci deliziavano» (J.W. GOETHE, Della mia vita. Poesia e verità, trad. it., 2 voll., Torino, UTET, 1957, vol. II, p. 650).

(18) A. Manzoni, Lettre à M. Chauvet, in Tutte le opere di Alessandro Manzoni, a cura di A. CHIARI e F. Ghisalberti, 7 voll. (1954-1991), Milano, Mondadori, vol. V, tomo III, Scritti linguistici e letterari, a cura di C. RicCARDI e B. TRAVI, 1991, pp. 154-155; corsivi miei.

(19) D. Diderot, Entretiens sur le Fils naturel, in Euvres de Denis Diderot, cit., vol. IV, Théâtre, p. 151 (cfr. Euvres esthétiques, a cura di P. VerniĖRE, Paris, Garnier, 1994, p. 118); corsivi miei.

(20) ID., De la poésie dramatique, in Euvres de Denis Diderot, cit., pp. 503-504 (ed. Garnier, p. 263); corsivi miei. 
Pourra-t-on raisonnablement lui [si riferisce al poeta] reprocher de n'avoir pas assez ménagé la délicatesse du spectateur? Je crois qu'on a imposé trop d'égards aux poëtes pour cette susceptibilité du public; qu'on leur a trop fait un devoir d'éviter tout ce qui pouvait déplaire ${ }^{21}$.

Sembra quindi nascere da uno spunto diderotiano la tendenza di Manzoni a scuotere i meccanismi cognitivi del pubblico irrigiditi dall'aspettativa nella conformità dell'opera a regole prescritte. Così come fonte di ispirazione per il suo immaginario può essere stata l'infrazione del codice classico costituita dalla nozione di sublime dal basso elaborata dagli Entretiens sur le Fils naturel, che opponevano alla suddivisione tradizionale dei generi un rimescolamento delle categorie di tragico e comico ${ }^{22}$. Soprattutto quando, nell'abolizione di confini rigidi fra i generi, Diderot si rivolgeva a Shakespeare, anticipando il Romanticismo:

Les tragédies de Shakespeare sont moitié vers et moitié prose. Le premier poëte qui nous fit rire avec de la prose, introduisit la prose dans la comédie. Le premier poëte qui nous fera pleurer avec de la prose, introduira la prose dans la tragédie. ${ }^{23}$

Nel definire il proprio combattuto apprezzamento del «mélange du comique et du sérieux» shakespeariano, alla cui formulazione contribuì, secondo Ezio Raimondi, la frequentazione del milieu degli idéologues ${ }^{24}$, Manzoni può forse aver avuto presente anche il pensiero di Diderot. In ogni caso l'elaborazione che lo conduce dalle tragedie ai Promessi Sposi ha seguito un itinerario simmetrico a quello del predecessore, muovendosi dal tentativo di introdurre il «mélange du plaisant et du sérieux» all'interno del genere drammatico e riuscendo poi alla consapevolezza che solo il «genre narratif» può assicurare una naturale compenetrazione di elementi comici e tragici:

Ne relit-on pas tous les jours des ouvrages dans le genre narratif, il est vrai, mais des ouvrages où ce mélange se retrouve bien souvent, et sans qu'il ait été besoin de le justifier,

(21) A. Manzoni, Lettre à M. Chauvet, cit., p. 95; corsivi miei.

(22) «La comédie et la tragédie sont de tous les états; avec cette différence, que la douleur et les larmes sont encore plus souvent sous les toits des sujets, que l'enjouement et la gaîté dans les palais des rois. C'est moins le sujet qui rend une pièce comique, sérieuse ou tragique, que le ton, les passions, les caractères et l'interrêt»; D. DIDEROT, Entretiens sur le Fils naturel, cit., pp. 179-190 (ed. Garnier, pp. 141-149).

(23) Ibid., p. 155 (ed. Garnier, p. 120). Si può ritenere Diderot (insieme a Lessing) un precursore dell'attenzione all'opera shakespeariana che animerà il romanticismo francese e tedesco. I riferimenti a Shakespeare si infittiscono nel Paradoxe sur le comédien (che non comparve nell'edizione Naigeon, ma fu pubblicato postumo nel 1831, dopo essere uscito in un primo abbozzo sulla «Correspondance littéraire»), che critica fortemente il dettato ampolloso delle tragedie di Corneille e Racine, opponendo loro la naturalezza di Shakespeare e avanzandone l'esempio come antidoto alla regola della «bienséance» (il cui superamento, si è visto, rappresenta un importante punto di contatto tra Diderot e Manzoni). Ispiratosi inizialmente a Voltaire, che nelle Lettres anglaises aveva esaltato Shakespeare, Diderot non lo segue poi nella successiva condanna del carattere "barbaro" delle sue tragedie (cfr. Y. BELAVAL, Diderot e la riforma del teatro, postfazione a D. DiDERot, Paradosso sull'attore, trad. it, Milano, Abscondita, 2002, pp. 87-101 e C. DEDEYAN, Diderot et la pensée anglaise, Firenze, Olschki, 1987, pp. 121-127).

(24) L'importanza della lettura di Shakespeare nel passaggio dal teatro al romanzo di vocazione realista è ribadita da Ezio RAIMONDI, che indica la centralità della Vie de Shakespeare di Guizot inviata a Manzoni da Fauriel (cfr. il suo Il dramma, il comico, il tragico, in ID., Il romanzo senza idillio, Torino, Einaudi, 1974, pp. 79-123). La prospettiva è ripresa da Luciano Bottoni, che così conclude il suo intervento sulla poetica teatrale del romanticismo lombardo: «La potenzialità paradigmatica del modello shakespeariano, la totalità analogica della sua mimesis, all'interno della Lettre à $M$. Chauvet avrebbe trasformato il programma di una drammaturgia epico-moralistica [...] in un'estetica embrionale del realismo romanzesco» (cfr. L. BOTTONI, Drammaturgia e sistemi letterari nel Conciliatore, in «Lettere Italiane», LI, 1983, p. 79). Si è poi tentato di estendere l'influenza shakespeariana su Manzoni anche alla teorizzazione estetica e alla poetica del coro (cfr. C. ANNONI, Lo spettacolo dell'uomo interiore, Milano, Vita e Pensiero, 1997, pp. 114-129). 
parce qu'il est tellement fondu dans la vérité entrainante de l'ensemble, que personne ne l'a remarqué pour en faire un sujet de censure? [...]

Mais [...] le mélange du plaisant et du sérieux pourra-t-il être transporté heureusement dans le genre dramatique d'une manière stable, et dans des ouvrages qui ne soient pas une exception? C'est, encore une fois, ce que je n'ose pas savoir ${ }^{25}$.

Manzoni segue Diderot nel decretare, a partire dall'esempio di Shakespeare, l'esigenza di contaminazione di basso e sublime, di tragedia e commedia, e soprattutto nell'intuire che tale trasformazione nella codificazione tradizionale dei generi poteva realizzarsi solo a patto di risolversi nell'osmosi di prosa e poesia ${ }^{26}$. Il modello diderotiano dimostrava che il rifiuto delle regole e delle suddivisioni di genere non poteva che causare l'abbandono del genere drammatico a favore del genre narratif.

Non sarà sfuggito infatti a Manzoni come l'impulso del philosophe a intraprendere la via del conte nascesse proprio a partire dal bisogno di continua giustificazione e integrazione del testo teatrale:

De cette portion d'une farce [l'Amico sincero di Goldoni] en trois actes, j'en fis la comédie du Fils naturel en cinq; et mon dessein n'étant pas de donner cet ouvrage au théâtre, j’y joignis quelques idées que j'avois sur la poëtique, la musique, la déclamation, et la pantomime; et je formai du tout une espèce de roman que j'intitulai Le fils naturel, ou Les Éprenves de la vertu, avec l'histoire véritable de la pièce ${ }^{27}$.

«Idées» e tensione all' «histoire véritable» provocano l'esplosione della struttura drammatica, che confluisce nel macchinario più indefinito dell'«espèce de roman». Diderot rivela che ciò che stiamo leggendo in qualità di dramma è già un romanzo, che allegare riflessioni teoriche dialogate a un'opera teatrale, entrando in prima persona come $M o i$, è scrivere un romanzo ${ }^{28}$. Il gesto di “uscire fuori dall'opera" per spiegarne i fondamenti è riconosciuto come un'operazione inevitabile per la dimostrazione della verità del proprio oggetto anche da Manzoni, che nel trattato Del romanzo storico ne illustra le dinamiche a proposito dell'Enriade di Voltaire. L'opera è posta a esempio di un genere costretto a trascendere se stesso, poiché il poeta, non potendo dimostrare all'interno del poema la verosimiglianza dei contenuti, è «ridotto a uscir-

(25) A. Manzoni, Lettre à M. Chauvet, cit., p. 102; corsivi miei.

(26) Anche se Diderot difende la pregnanza estetica delle unità aristoteliche, la sua drammaturgia istituisce una divaricazione dal teatro classico che coinvolge, oltre all'ambito stilistico e tematico, anche quello formale: «Nous sommes loin non seulement des principes stylistiques de la tragédie classique régie par l'idéal de la bienséance, mais encore de ses principes formels, car dans la tragédie classique la réalité n'existe que dans la mesure où elle est devenue langage» (cfr. P. SzONDI, Denis Diderot: théorie et pratique dramatique, in Diderot et le théâtre, cit., pp. 40-41).

(27) D. DidERot, De la poésie dramatique, cit., p. 456 (ed. Garnier, pp. 222-223). Jean Starobinski ha definito le due opere diderotiane di teoria drammatica attraverso la formula dell'«hybride esthétique», ritenendole composizioni indefinibili e contraddittorie che unicamente il romanzo, genere dalle frontiere non delimitate, è in grado di contenere (cfr. J. Starobinski, L'accent de la vérité, in Diderot et le théâtre, cit., pp. 9-28).
(28) Per un'analisi delle interferenze fra teatro e romanzo nell'estetica diderotiana, in senso inverso ma complementare a quello qui considerato, cfr. P. FRANTZ, Le roman, perte ou salut du théatre?, in Il romanzo a teatro. Atti del Convegno Internazionale della Società Universitaria per gli Studi di Lingua e Letteratura Francese, Verona, 11-13 novembre 2004, a cura di F. PIVA, Fasano, Schena, 2005, pp. 37-48. La capacità diderotiana di stravolgere i generi tradizionali, mediante la mescolanza di narrazione e pensiero, è sottilmente illustrata da Pierre Hartmann: «le théoricien des monstres génétiques qu'est Diderot crée de toute pièce des monstres littéraires qu'il anime le temps nécessaire à l'exploration philosophique et au travail euristique, et qui se laissent si peu ranger dans les catégories de la poétique traditionelle que l'exégèse la plus moderne peine encore à les classer. [...] Au lieu de soumissionner la littérature à la philosophie, il investit le discours philosophique de la liberté exploratoire qui seule définit la grande littérature» (cfr. P. HARTMann, Diderot ou la figuration du philosophe, Paris, José Corti, 2003, p. 21). 
ne fuori, per asserir formalmente e provare e discutere, col mezzo di quella ch'egli chiamò più d'una volta la vile prosa $\gg^{29}$. Nell'analisi dell'opera volterriana, illustrata come primo campione del passaggio, destinato a sconvolgere i generi codificati, dalla poesia (seppur epica) alla prosa di dimostrazione e di discussione, Manzoni guarda anche alla propria esperienza di drammaturgo costretto a «puntellare» le strutture tragiche con argomentazioni teoriche ${ }^{30}$. La «vile prosa» adottata da Voltaire ricorda da vicino, nella rilettura manzoniana, l'«espèce de roman» di diderotiana memoria, prefigurando forse la prosa dei Promessi Sposi.

Tra i meccanismi che forzano la struttura del teatro manzoniano verso le regioni del romanzo, già anticipata nel teatro di Diderot è ad esempio la necessità di emersione della voce autoriale, che manifesta una tendenza a esorbitare rispetto all'oggettività del nucleo rappresentativo ${ }^{31}$. Nel paragrafo Des entr'actes della Poésie dramatique tale esigenza aveva spinto Diderot a teorizzare paradossali «scènes ébauchées», cioè «incidens que le poëte dérobe aux spectateurs, et qui supposent, dans l'intérieur de la maison, des entretiens entre ses personnages». L'opera teatrale prevedeva cioè la presenza di una zona preclusa allo sguardo dello spettatore, trasformando così lo spazio e il tempo della scena in dimensioni insufficienti all'espressione del poeta, appagata unicamente mediante una deviazione dalle modalità della rappresentazione a quelle della narrazione ${ }^{32}$. Lo stesso processo regolava la trascrizione della pantomima, «surcroît de plaisir» derivante dal «connoître le jeu, tel que le poëte l'a conçu» che si differenziava dal godimento estetico veicolato dalla visione diretta dell'azione scenica. Anzi la conoscenza da parte del pubblico di quel «tableau qui existoit dans l'imagination du poëte, lorsqu'il écrivoit; et qu'il voudroit que la scène montrât à chaque instant lorsqu'on le joue» instaurava nel fruitore un processo giudicante di confronto della recitazione e dello scenario immaginato dell'autore: «Le poëte vous dit: comparez ce jeu avec celui de vos acteurs; et jugez» ${ }^{33}$. Lo stesso valore di spiraglio sull'immaginazione del poeta sarà assunto dal coro manzoniano, concepito come

(29) A. MANZoni, Del romanzo storico e, in genere, de' componimenti misti di storia e d'invenzione, in Edizione nazionale ed europea delle opere di Alessandro Manzoni, cit., vol. 14 (2000), p. 45.

(30) L'ipotesi che la struttura delle due tragedie «avvolte dalle relative scritture storiche», così come si presenta nell'edizione delle opere curata dallo stesso Manzoni a partire dagli anni Quaranta, possa mimare la configurazione individuata nell'Enriade è stata avanzata da Gianfranco Contini, secondo il quale la poetica di commistione di materiali di invenzione e supporti di giustificazione storica, trasferita dal genere epico prescelto da Voltaire all'ambito teatrale e da qui a quello romanzesco, avrebbe costituito la prima motivazione del romanzo manzoniano, in seguito sostituita da un «nuovo fondamento» rintracciabile nel discorso Del romanzo storico, a superamento dell'iniziale concezione. Per il critico, quindi, la prima ideazione del romanzo si sarebbe sviluppata a partire dall'esperienza teatrale e dall'analisi delle trasformazioni attuate ai generi tradizionali dalle allegazioni della vile prosa, necessitate da una più pressante adesione al vero storico (cfr. G. CONTINI, Manzoni contro Manzoni (Un sasso in piccionaia), in ID., Pagine ticinesi, a cura di R. BROGGINI, Milano, Salvioni, 1986, pp. 214-221).

(31) Elena Sala di Felice ha individuato tra i mo- tivi che possono aver causato il passaggio di Manzoni al genere romanzesco, l'appartenenza della tragedia manzoniana alla categoria del «dramma moderno», genere caratterizzato, secondo la definizione di Peter Szondi, dall'affacciarsi di un io epico. Le modificazioni spaziali e temporali operate sull'organismo teatrale causano infatti una discontinuità nel tempo e una dilatazione dello spazio che necessitano dell'intervento unificante di un «montatore». L'io «ipertrofico» emerso nell'opera drammaturgica non è più contenibile nel «cantuccio» del coro e richiede un nuovo contenitore per esprimersi: il romanzo (cfr. E. SAla di FELICE, Manzoni e la (im) moralità del teatro, in «Intersezioni», VII, $\mathrm{n}$. 2, 1987, pp. 235-261 e P. SzONDI, Teoria del dramma moderno, trad. it., Torino, Einaudi, 1962).

(32) Negare allo sguardo dello spettatore di teatro una percezione d'insieme è azione fortemente antiaristotelica. In più luoghi, sia per la tragedia che per l'epica, la Poetica si vale della metafora dello sguardo d'insieme per definire l'unitarietà temporale del componimento: «si deve poter abbracciare con uno sguardo l'inizio e la fine» (ARISTOTELE, Poetica, trad. it., a cura di D. Lanza, Milano, Rizzoli, 1997 , cap. 24, p. 205; vedi anche cap. 7 e cap. 23).

(33) D. DiDEROT, De la poésie dramatique, cit., pp. 485-490 e pp. 551-553 (ed. Garnier, pp. 243248 e pp. 277-278). 
«personificazione dei pensieri morali che l'azione ispira» e «organo dei sentimenti del poeta» ${ }^{34}$. L'esigenza che impone a Manzoni l'interpolazione del coro risulta cioè simile a quella diderotiana di un diaframma morale e razionalizzante tra scena e pubblico, che svolga la funzione di chiarire l'intenzione dell'autore. Per dare modo al commento autoriale di dispiegarsi, la scrittura drammatica manzoniana, così come quella diderotiana, necessita spesso di apparati esplicativi esterni all'opera, a glossare continuamente il testo propriamente teatrale illustrandone le ragioni estetiche o storiche. In questo senso, le Notizie storiche apposte al Carmagnola e all'Adelchi presentano una funzione analoga agli Entretiens sur le Fils naturel o al trattato De la poésie dramatique. Manzoni e Diderot condividono la preoccupazione di accompagnare all'azione l'osservazione, che è sentita però come corpo estraneo e distaccato rispetto all'azione, in quanto fondamentalmente soggettiva e quindi rivela dall'interno stesso dell'opera teatrale l'instabilità del genere drammatico ${ }^{35}$.

L'unité delegittimata dalla drammaturgia manzoniana persiste nel Fermo e Lucia e nei Promessi Sposi come ideale negativo che il romanzo contesta, secondo dinamiche già rintracciabili nelle discussioni antiletterarie dei personaggi e dei narratori di Jacques le fataliste. Un artificio risalente alla teorica drammatica di fonte aristotelica che Manzoni evoca e subito delegittima, riprendendo stilemi tipici dell'apparato metanarrativo di Jacques le fataliste, è ad esempio la tecnica dell'incontro-agnizione:

Noi avremmo voluto che la nostra storia registrasse a questo passaggio qualche incontro, qualche avvenimento inaspettato, per poterne illustrare quel torrente [il Bione], e togliere il suo nome dalla oscurità; ma la storia non ne registra ${ }^{36}$.

Nell'allusione in negativo all'espediente dell'incontro, ha forse agito in Manzoni la memoria dei frequenti inserti diderotiani che prendono di mira la tendenza romanzesca a ridurre i rapporti tra i personaggi a un limitato numero di casi, organizzando incontri fortuiti tra personaggi già legati fra loro da relazioni intercorse precedentemente:

Et pourquoi le vieux militaire ne seroit-il pas ou le capitaine de Jacques ou le camarade de son capitaine? - Mais il est mort. - Vous le croyez?... Pourquoi la jeune paysanne ne seroit-elle

(34) A. Manzoni, Prefazione al Carmagnola, in ID., Scritti di teoria letteraria, a cura di A. SozzI CASANOVA, Milano, Rizzoli, 1981, p. 50.

(35) Nel passaggio dal teatro al romanzo il livello dell'osservazione sarà mantenuto per entrambi da quel piano di discorso sfasato rispetto al procedere della narrazione costituito dalla digressione, in cui è svolta la comunicazione tra autore e fruitore già sperimentata per il genere teatrale. Secondo Giovanni Macchia, il coro «era già una delle forme di riposo nello svolgimento dell'azione su cui come romanziere [Manzoni] avrebbe tanto lavorato. Era un tentativo di digressione», mostrava come si rendesse «necessaria un'altra forma d'espressione, che non dimenticasse del tutto l'esperienza della tragedia»: «ciò che era lo statismo [...] nell'antica tragedia greca, lirica nel tono e nelle espressioni, e che divideva un episodio dall'altro, produceva nel romanzo uno stacco fondamentale, quasi diventando una forma di intermezzo, del tutto al di fuori dello svolgimento dell'azione» (G. MACCHIA, Nascita e morte della digressione, in ID., Tra don
Giovanni e don Rodrigo. Scenari secenteschi, Milano, Adelphi, 1989, p. 22). In seguito Ezio Raimondi notava l'evoluzione della «voce epica del coro» nella «presenza costante e sinuosa di un personaggio multiplo, non vincolato alla sola tonalità del linguaggio lirico» (E. RAImONDI, Il dramma, la storia, in ID., Romanticismo italiano e romanticismo europeo, Milano, Bruno Mondadori, 1997, pp. 92-111). Anche Diderot tocca tangenzialmente il problema, addebitando proprio alla mancanza del coro la necessità di servirsi, nei drammi moderni, delle «scènes simultanées» e di personaggi secondari che agiscano in concomitanza alla scena principale: «La ressource de ces personnages nous est d'autant plus nécessaire, que, privés des chœurs qui représentoient le peuple dans les drames anciens, nos pièces, renfermées dans l'intérieur de nos habitations, manquent, pour ainsi dire, d'un fond sur lequel les figures sont projetées»; D. DIDEROT, De la poésie dramatique, cit., p. 496 (ed. Garnier, p. 251).

(36) FL, II, VIII, pp. 300-301. 
pas ou la dame Suzon, ou la dame Marguerite, ou l'hôtesse du Grand-Cerf, ou la mère Jeanne, ou même Denise sa fille? Un faiseur de roman n'y manqueroit pas, mais je n'aime pas les romans, à moins que ce ne soient ceux de Richardson. Je fais l'histoire; cette histoire intéressera ou n'intéressera pas, c'est le moindre de mes soucis. Mon projet est d'être vrai, je l'ai rempli. Ainsi, je ne ferai point revenir frère Jean de Lisbonne; ce gros prieur qui vient à nous dans un cabriolet, à côté d'une jeune et jolie femme, ce ne sera point l'abbé Hudson ${ }^{37}$.

L'organicità dell'opera, prescritta dal dettato aristotelico e ripresa dai faiseurs de romans, è rifiutata: nessun legame arbitrario organizza gli incontri di Jacques le fataliste, così come nessun incontro connette l'esistenza storica del Bione agli eventi romanzeschi, ricomponendo in ordine l'«oscurità» informe della storia.

Altro meccanismo appartenente al formulario della tragedia classica, confutato da Diderot prima e da Manzoni poi, è la premonizione. Se l'agnizione, intesa in senso aristotelico, riconduce a unità la molteplicità dei personaggi, il presagio conferisce compiutezza alla successione temporale, individuando corrispondenze fra eventi logicamente lontani. La derisione delle premonizioni di Jacques operata dall'illuminista Diderot riecheggia nell'episodio manzoniano del commiato di padre Cristoforo, Renzo e Lucia, con la differenza che Manzoni reinterpreta la sua fonte recuperando per le intuizioni del cuore una validità cognitiva assente nell'antecedente ${ }^{38}$. «Certo il cuore, chi gli dà retta, ha sempre qualche cosa da dire su quello che sarà. Ma che sa il cuore? Appena un poco di quello che è già accaduto», replica il narratore manzoniano commentando il presagio di Cristoforo («il cuor mi dice che ci rivedremo presto»); per poi orchestrare gli eventi, però, in maniera antitetica a tale sentenza ${ }^{39}$. Diderot smentiva analogamente la sensazione premonitrice di Jacques (il quale, osservando la forza attrattiva esercitata dai patiboli sul suo cavallo, inferiva per sé un destino di morte: «Qu'est-ce que cela signifie? [...]. Est-ce un advertissement du destin?») esibendo il dato di fatto dell'appartenenza del cavallo al boia. Nullificate sono in maniera simile nei due episodi le preconizzazioni dei personaggi, in modo che il tempo della vicenda non conosca i ritorni e le simmetrie che caratterizzano la dimensione temporale del teatro classico, in cui spesso lo svolgimento avvera i presagi conosciuti fin dall'inizio.

Il procedimento che più di ogni altro imprime alle vicende dei romanzi un andamento unitario è l'abuso della tematica amorosa, secondo lo stesso Manzoni della Lettre à $M$. Chauvet esito estremo della semplificazione spazio-temporale causata dalle unità aristoteliche. L'argomentazione della Lettre coincide infatti con i ragionamenti della Digressione che, nel Fermo e Lucia, rifiutano l'amore come tema degno di narrazione $^{40}$. Analogo soprattutto il riferimento agli «altri sentimenti dei quali il mondo ha bisogno» come nuovi soggetti da sostituire alle vicende d'amore in controtendenza rispetto alla tradizione romanzesca ${ }^{41}$, che richiama da vicino la rivendicazione delle «variétés infinies» e delle «combinaisons singulières» ${ }^{42}$ di sentimento operata in sede di estetica teatrale. Come fonte della Digressione, già Giovanni Getto poneva la polemica antiamorosa di Jacques le fataliste:

Ainsi je vous conseille, lecteur, de prendre votre parti; et au défaut des amours de Jacques, de vous accommoder des aventures du secrétaire du marquis des Arcis. [...] Et puis,

(37) JF, p. 358 (ed. Laffont, p. 883); corsivi miei.

(38) Cfr. il commento alla premonizione di fra Cristoforo di Giorgio Ficara (Sentimento e ragione, approfondimento critico al capitolo VIII, in A. Manzoni, I Promessi Sposi, Torino, Petrini, 1986, pp. 153-158).

(39) A. Manzoni, Promessi Sposi, a cura di S. S. Nigro, Milano, Mondadori, VIII, p. 162 (d'ora in poi $P S)$.

(40) Sulla Digressione del Fermo, cfr. S.S. Nigro, La tabacchiera di don Lisander. Saggio sui "Promessi Sposi”, Torino, Einaudi, 1996, cap. I, pp. 15-37.

(41) FL, II, I, p. 173.

(42) A. Manzoni, Lettre à M. Chauvet, cit., p. 133. 
lecteur, toujours des contes d'amour; un, deux, trois quatre contes d'amour que je vous ai faits; trois ou quatre autres contes d'amour qui vous reviennent encore: ce sont beaucoup de contes d'amour. [...] Toutes vos nouvelles en vers ou en prose sont des contes d'amour; presque tous vos poëmes, élégies, églogues, idyilles, chansons, épîtres, comédies, tragédies, opéras, sont des contes d'amours. Presque toutes vos peintures et sculptures ne sont que des contes d'amour. Vous êtes aux contes d'amour pour toute nourriture depuis que vous existez, et vous ne vous en lassez point. L'on vous tient à ce régime et l'on vous y tiendra long-temps encore, hommes et femmes, grands et petits enfans, sans que vous vous en lassiez. En vérité, cela est merveilleux. Je voudrois que l'histoire du secrétaire du marquis des Arcis fût encore un conte d'amour; mais j'ai peur qu'il n'en soit rien, et que vous n'en soyez ennuyé. Tant pis pour le marquis des Arcis, pour le maître de Jacques, pour vous, lecteur, et pour moi ${ }^{43}$.

Che ogni evento sia ricondotto a una storia d'amore è un voler essere del lettore, che l'autore tende a frustrare intraprendendo in tutto il romanzo una continua dilazione del conte d'amour, retaggio di una tradizione letteraria compendiata ironicamente in quell'elencatio asindetica di generi fortemente codificati tra cui spiccano in chiusura le forme teatrali. Il tono di sfida alle abitudini percettive del lettore, che costituiscono il fondamento delle regole, è soprattutto ciò che dell'inserzione di Jacques riemerge nel colloquio del narratore del Fermo con il suo «personaggio ideale». Così nell'allusione diderotiana all'ennui del lettore si intravede già ciò che in Manzoni sarà il rifiuto del suo divertimento. Simili inoltre la scelta di oblazione della tematica amorosa e la sua motivazione estetica: l'amore deve essere negato al lettore perché costituisce la manifestazione ultima di un'esigenza di armonizzazione dei sentimenti che deriva dalle regole imposte dalla convenzione letteraria. Di nuovo si nota quella sovrapposizione di rifiuto delle convenzioni ed estetica romanzesca che sembra derivare da Diderot soprattutto quando si risolve nel porre le consuetudini del genere teatrale come modello negativo per il romanzo. E quando giunge alla conclusione che l'opposizione all'eccesso d'amore nelle trame teatrali conduca alla necessità di ricognizione di ciò che non si riferisce alle dinamiche amorose e che la tassonomia delle passioni del teatro non contemplava, quel «fond du cœur» rivendicato come orizzonte privilegiato della narrazione dall'ostessa di Jacques le fataliste.

Se ricondotte all'esempio diderotiano, anche le riflessioni metanarrative manzoniane che giocano sull'autonomia dei personaggi assumono una funzione di rottura contro le prescrizioni della tradizione letteraria che costringono l'autore a conchiudere il proprio soggetto entro forme prestabilite. Nel sancire la natura non codificabile dell'animo umano al fine di dimostrare l'impossibilità di una definizione unitaria del mondo da parte della scrittura, Manzoni riprende Diderot quasi alla lettera. Eco dell'insufficienza del narratore di Jacques le fataliste davanti al proprio personaggio ( Je ne sais s'il [Jacques] commença par abaisser les jupons ou par dégager le pied»; «Nos deux voyageurs n'étoient point suivis: j'ignore ce qui se passa dans l'auberge après leur départ $\gg^{44}$ ) è ad esempio il non-sapere ${ }^{45}$ esperito dal narratore manzoniano di fronte al vicario di provvisione ( «Del resto, quel che facesse precisamente non si può sapere, giacchè era solo; e la storia è costretta a indovinare. Fortuna che c'è avvezza») o a Renzo («Come la facesse quando trovava due strade; se quella poca pratica, con quel poco barlume, fossero quelli che l'aiutassero a trovar sempre la buona, o se l'in-

(43) JF, pp. 269-270 (ed. Laffont, p. 841); corsivi miei.

(44) Ibid., pp. 7 e 20 (ed. Laffont, pp. 715 e 722).

(45) Per la categoria del non-sapere come strut- tura fondamentale del dettato manzoniano, cfr. S. Agosti, Per una semiologia della voce narrativa nei "Promessi Sposi", in ID., Enunciazione e racconto. Per una semiologia della voce narrativa, Bologna, il Mulino, 1989, pp. 107-153. 
dovinasse sempre alla ventura, non ve lo saprei dire»). Oppure la provenienza indefinita di alcune battute che i Promessi Sposi registrano pur non essendo attribuibili ai legittimi proprietari. Come un’idea del conte zio («Così anche noi non sapremmo dire se dal fondo naturale del suo cervello, o dall'insinuazione d'Attilio, venisse al conte zio la risoluzione di servirsi del padre provinciale per troncare nella miglior maniera quel nodo imbrogliato ${ }^{46}$ ), variante manzoniana delle riflessioni sull'inconsistenza dei giuramenti che figurano in Jacques le fataliste, senza appartenere precisamente a nessuno: «Je ne sais de qui sont ces réflexions, de Jacques, de son maître ou de moi, il est certain qu'elles sont de l'un des trois» ${ }^{47}$.

All'unità di ruolo dei personaggi tragici, si sostituisce nei Promessi Sposi la problematizzazione dello sguardo autoriale sull'interiorità dei protagonisti, che genera indecisioni nella lineare spiegazione degli eventi, trasformandosi in domanda inconcludibile che rimbalza dall'anonimo al narratore moderno fino alla coscienza del lettore, coinvolto nella riflessione sulle ragioni occulte del comportamento dei personaggi in apparenza più limpidi:

In mezzo a quella sua gran collera, aveva Renzo pensato di che profitto poteva esser per lui lo spavento di Lucia? E non aveva adoperato un po' d'artifizio a farlo crescere, per farlo fruttare? Il nostro autore protesta di non ne saper nulla; e io credo che nemmeno Renzo non lo sapesse bene. [...] Qui l'autore confessa di non sapere un'altra cosa: se Lucia fosse, in tutto e per tutto, malcontenta d'esser stata spinta ad acconsentire. Noi lasciamo, come lui, la cosa in dubbio ${ }^{48}$.

Un dubbio dalla natura molto simile agitava già il narratore diderotiano, che perplesso a proposito della reale consistenza dell'anima dei suoi personaggi manteneva irrisolta ogni ipotesi nel consegnarla al lettore:

Quoique Jacques, la meilleure pâte d'homme qu'on puisse imaginer, fût tendrement attaché à son maître, je voudrois bien savoir ce qui se passa au fond de son âme, si-non dans le premier moment, du-moins lorsqu'il fut bien assuré que cette chûte n'auroit point de suite fâcheuse, et s'il put se refuser à un léger mouvement de joie secrète d'un accident qui apprendroit à son maître ce que c'étoit qu'une blessure au genou. Une autre chose, lecteur, que je voudrois bien que vous me dissiez, c'est si son maître n'eût pas mieux aimé être blessé, même un peu plus grièvement, ailleurs qu'au genou, ou s'il ne fut pas plus sensible à la honte qu'à la douleur ${ }^{49}$.

In entrambi i casi le modalità di relazione di una coppia di personaggi comprendono rapporti molto più articolati di quelli che la narrazione è in grado di porre in luce. La scrittura denuncia l'esistenza di motivazioni dell'agire umano talmente sature di componenti eterogenee da non risultare completamente ricostruibili sulla pagina.

(46) PS, XIII, p. 254, XXXVII, p. 715 e XIX, p. 561. Hermann Grosser, esaminando i movimenti di avvicinamento e allontanamento del punto di vista del narratore dai punti di vista dei personaggi, si limita a leggere il passo come denuncia dell'impossibilità di interpretare gli atti e i pensieri dei personaggi storici. Tuttavia, l'ironia sui procedimenti dell'introspezione coinvolge, come si vedrà, anche un personaggio di fantasia come Renzo, che invece Grosser considera un punto di osservazione dalla liceità indiscutibile (cfr. il suo Osservazioni sulla tecnica narrativa e sullo stile dei "Promessi Sposi", in «Giornale storico della letteratura italiana», vol. CLVIII, 1981, pp. 409-440).

(47) JF, p. 169 (ed. Laffont, p. 794). Cfr. anche: «Lecteur, il me vient un scrupule, c'est d'avoir fait honneur à Jacques ou à son maître de quelques réflexions qui vous appartiennent de droit; si cela est, vous pouvez les reprendre sans qu'ils s'en formalisent»; ibid., p. 314 (ed. Laffont, p. 862).

(48) PS, VII, p. 122.

(49) JF, p. 25 (ed. Laffont, p. 724). 
Le sequenze ostinate di interrogative che affollano le opere diderotiane, come nel lungo commento al comportamento di Mlle d'Aisnon:

Est-ce que cette fille comprit rien aux artifices de la dame de La Pommeraye avant le dénouement? Est-ce qu'elle n'auroit pas mieux aimé accepter les offres que la main du marquis, et l'avoir pour amant que pour époux? Est-ce qu'elle n'était pas continuellement sous les menaces et le despotisme de la marquise? Peut-on la blâmer de son horrible aversion pour un état infâme? Et si l'on prend le parti de l'en estimer davantage, peut-on exiger d'elle bien de la délicatesse, bien du scrupule dans le choix des moyens de s'en tirer? ${ }^{50}$

\section{sono rintracciabili lungo la pagina manzoniana in disposizione quasi invariata:}

Quell'uomo era stato a sentire all'uscio del suo padrone: aveva fatto bene? E fra Cristoforo faceva bene a lodarlo di ciò? Secondo le regole più comuni e men contraddette, è cosa molto brutta; ma quel caso non poteva riguardarsi come un'eccezione? E ci sono delle eccezioni alle regole più comuni e men contraddette? Questioni importanti; ma che il lettore risolverà da sé, se ne ha voglia. Noi non intendiamo di dar giudizi: ci basta aver dei fatti da raccontare ${ }^{51}$.

La possibilità di «eccezioni» a prescrizioni morali univocamente intese incrina la compattezza e la compiutezza della coscienza dei personaggi: $\mathrm{i}$ «fatti», le cause e gli effetti visibili dei comportamenti sono percorsi da ambiguità sotterranee che dischiudono possibili, inquietanti «giudizi», sollecitati e subito negati dalla scrittura. Manzoni ricalca da Diderot un meccanismo di relativizzazione del punto di vista dell'osservatore che mina le certezze conoscitive del lettore, rieducandolo così all'analisi critica dell'univocità dei personaggi romanzeschi. Sottostà alla critica delle categorizzazioni morali univoche il rifiuto di regole di composizione che costringano alla semplificazione dell'interiorità dei personaggi e presentino al fruitore un oggetto letterario già codificato. Il rifiuto della règle teorizzato in sede di estetica teatrale e ribadito negli inserti metaletterari della scrittura romanzesca si traduce operativamente nel tentativo di ritrarre in maniera più veridica l'oscillazione del «fond du coeur», che la regola non permetteva di restituire nella sua complessità.

Il romanzo è quindi prescelto da Manzoni, sulla scia di Diderot, in quanto la sua struttura aperta può consentire il superamento delle convenzioni letterarie imposte in maniera rigida al testo teatrale, in direzione di una maggiore aderenza a un contenuto di verità che si percepisce come disarmonico e disomogeneo. Ma a tradire un'ascendenza diderotiana è ancora la consapevolezza che sia inevitabile anche per il romanzo, in quanto opera di invenzione, operare una semplificazione arbitraria del reale. Il romanzo manzoniano denuncia perciò fin dal primo abbozzo, con movimento mistificatorio che di nuovo sembra ispirarsi alle mistifications diderotiane ${ }^{52}$, la sua

(50) Simili interrogativi possono essere sollevati specularmente anche nell'analisi delle azioni di Mme de la Pommeraye («Et vous croyez, lecteur, que l'apologie de Mme de La Pommeraye et plus difficile à faire?») e si risolvono spesso in ammonimento allo sbilanciamento frettoloso di giudizio del lettore: «Ah! Lecteur, vous êtes bien léger dans vos éloges, et bien sévère dans votre blâme», ibid., pp. 238-241 (ed. Laffont, pp. 826-827).

(51) PS, VI, p. 108.

(52) L'architettura romanzesca manzoniana contiene in sé la sua stessa negazione critica, è uno svolgimento che contravviene alle sue stesse premesse.
Anche la conclusione dei Promessi Sposi rappresenta in certo qual modo l'avverarsi della premonizione di padre Cristoforo e la sconfessione della conseguente precisazione ironica del narratore. $\mathrm{La}$ costituzione di una struttura che continuamente costruisce e distrugge se stessa, secondo una strategia di contemporanea adozione e negazione del romanzesco, è ancora una volta un'eredità diderotiana. Come I Promessi Sposi infatti, anche Jacques le fataliste si concludeva con l'incontro fortuito di Jacques e del padrone in un castello (luogo tipicamente romanesque, già preso di mira dall'ironia mistificante del narratore) e la soluzione felice degli 
appartenenza allo stesso orizzonte "teatrale" da cui sembrava allontanarsi e al contrario la sua alterità rispetto alla dimensione della «vita reale» cui invece intendeva ispirarsi, arrivando a tacciare di irrealtà il suo stesso finale: «la morte e il matrimonio terminano per lo più le tragedie e le commedie del teatro, ma danno sovente principio alle tragedie e alle commedie della vita reale» ${ }^{53}$.

BIANCA GAI

amori di Jacques, cioè con un lieto fine in contraddizione con le premesse d'avvio, che citavano tra le variabili evenemenziali scartate nella composizione del romanzo, in quanto appartenenti alla dimensione irreale del conte, avvenimenti del tutto sovrapponibili ai fatti narrati in explicit: «Qu'est- ce qui m'empêcheroit de marier le maître et de le faire cocu? D'embarquer Jacques pour les îles? D'y conduire son maître? De les ramener tous les deux en France sur le même vaisseau? Qu'il est facile de faire des contes!», JF, pp. 5-6 (ed. Laffont, p. 714). (53) FL, IV, 1, p. 621. 